\title{
A risk-predictive model for invasive pulmonary aspergillosis in patients with acute exacerbation of chronic obstructive pulmonary disease
}

\author{
Yu Gu${ }^{1+}\left(\mathbb{0}\right.$, Xianping Ye ${ }^{1 \dagger}$, Yuxiu Liu ${ }^{2 \dagger}$, Yu Wang ${ }^{3}$, Kunlu Shen ${ }^{4}$, Jinjin Zhong ${ }^{3}$, Bilin Chen ${ }^{3}$ and Xin Su $1,3,4^{*}$
}

\begin{abstract}
Objectives: Invasive pulmonary aspergillosis (IPA) is increasingly reported in chronic obstructive pulmonary disease (COPD) patients. These patients often have poor clinical outcomes. Early recognition of IPA in COPD is always challenging. We aimed to develop and validate a risk model using readily available clinical parameters to predict IPA for acute exacerbation of COPD (AECOPD) patients.

Methods: We performed a retrospective cohort study. AECOPD patients who were admitted to Jinling Hospital between January 2012 and December 2017 were included. 880 AECOPD patients were randomly divided into the training set $(70 \%, n=616)$ and validation set $(30 \%, n=264)$. A nomogram model was developed using multivariate logistic regression from training set. The discrimination and calibration of model were validated internally. Decision curve analyses assessed the clinical utility of the nomogram.
\end{abstract}

Results: The incidence of IPA in hospitalized AECOPD patients was 9.6\% in the training set (59 cases of IPA) and 9.1\% in the validation set (24 cases of IPA), respectively. The nomogram model consisted of independent factors associated with IPA included lung function GOLD III-IV, use of broad-spectrum antibiotic over 10 days in the last month, oral or intravenous corticosteroids (prednisone) over $265 \mathrm{mg}$ in the last 3 months and serum albumin $<30 \mathrm{~g} / \mathrm{L}$. The model performed good discrimination and calibration in validation set (c-statistic, 0.79 [95\% Cl 0.68-0.90]). The 95\% $\mathrm{Cl}$ region of calibration belt did not cross the 45-degree diagonal bisector line $(P=0.887)$.

Conclusion: The simple risk predictive model for earlier recognition of IPA is useful in hospitalized AECOPD patients. Keywords: IPA, AECOPD, Risk factors, Nomogram, Model

\section{Background}

Nowadays, chronic obstructive pulmonary disease (COPD) has been widely recognized as a major risk factor for invasive pulmonary aspergillosis (IPA) [1]. The current mortality rate might be as high as $100 \%$ in

\footnotetext{
*Correspondence: suxinjs@163.com

${ }^{\dagger}$ Yu Gu, Xianping Ye and Yuxiu Liu contributed equally to this study 1 Department of Respiratory and Critical Care Medicine, Jinling Hospital, Nanjing Medical University, Nanjing 210002, China

Full list of author information is available at the end of the article
}

untreated patients [2,3]. Delayed diagnosis or delayed antifungal therapy are associated with increased mortality in patients with IPA $[4,5]$. Early recognition of IPA represents an opportunity to improve clinical outcome. In fact, the diagnosis of IPA in COPD patients is difficult. One of the important reasons is that the clinical manifestations and imaging presentations are not specific. On the other hand, the current laboratory diagnostic tests are not very sensitive for non-neutropenic population

c) The Author(s) 2021. This article is licensed under a Creative Commons Attribution 4.0 International License, which permits use, sharing, adaptation, distribution and reproduction in any medium or format, as long as you give appropriate credit to the original author(s) and the source, provide a link to the Creative Commons licence, and indicate if changes were made. The images or other third party material in this article are included in the article's Creative Commons licence, unless indicated otherwise in a credit line to the material. If material is not included in the article's Creative Commons licence and your intended use is not permitted by statutory regulation or exceeds the permitted use, you will need to obtain permission directly from the copyright holder. To view a copy of this licence, visit http://creativecommons.org/licenses/by/4.0/. The Creative Commons Public Domain Dedication waiver (http://creativecommons.org/publicdomain/zero/1.0/) applies to the data made available in this article, unless otherwise stated in a credit line to the data. 
including COPD patients, leading to delays in diagnosis and treatment of IPA $[4,6,7]$.

Combination of risk factors, clinical manifestations, and laboratory tests results together, is the current strategy for IPA diagnosis. Fully revealing risk factors of IPA in COPD patients is important to help clinicians identify infection [8]. In recent years, it has been revealed that worse lung function (GOLD III or IV) and systematic usage of corticosteroid play a significant role in the development of IPA [1]. Studies have also shown that COPD patients who were admitted to ICU, had chronic heart failure, receiving antibiotic treatment longer than 10 days in the past 3 months are independent predictors of IPA in COPD patients [3, 9]. The identification of one or more predisposing conditions would be critical to trigger further diagnostic exploration, which is a benefit to the early diagnosis and treatment.

There are multiple risk factors of IPA in COPD patients. But, few studies focused on the weight of each related risk factors. There is no Risk-Predictive scoring model of IPA currently. Therefore, it is necessary to further investigate the risk factors of IPA in COPD patients. It is of great importance for early diagnosis and treatment.

This study aims to identify the risk factors for IPA in hospitalized acute exacerbation of COPD (AECOPD) patients. Then, we develop and validate a risk-prediction model for rapid recognition and appropriate empirical antifungal treatment in severe AECOPD patients, especially in source limited hospitals.

\section{Methods}

\section{Data source}

This retrospective single-center study was based on the clinical records of AECOPD patients retrieved from the Department of Respiratory and Critical Care Medicine of Jinling Hospital from January 2012 to December 2017.

Training set and validation set. AECOPD patients fulfill with study design were enrolled into our study. We randomly assigned $70 \%$ cases into training set, and 30\% cases into internal validation set.

\section{Participants}

We included patients with AECOPD needing hospitalization. The exclusion criteria were as follows: (1) Patients suffering from neutropenia (peripheral blood absolute neutrophils count less than $0.5 \times 109 / \mathrm{L}$ ) or with hematological malignancy; (2) patients admitted to hospital without AECOPD; (3) patients with insufficient information. The diagnosis of AECOPD was based on the Global Initiative for Chronic Obstructive Lung Disease (GOLD) guidelines [10]. Bulpa criteria was applied to IPA diagnosis [1]. Therefore, Proven IPA was diagnosed by existence of mycelium and related tissue damage in histopathological examination of lung tissue and was accompanied by any of the following: (1) isolation of Aspergillus in the lower respiratory tract (LRT) samples; (2) positive serum Aspergillus antigen or antibody; (3) direct molecular immunology or culture methods observed that the mycelium was Aspergillus filaments. Probable IPA was diagnosed by the coexistence of host factors (severe COPD patients according to GOLD stage and usually treated by steroids), clinical manifestations (COPD patients had a recent exacerbation of dyspnea and suggestive chest imaging, and poor response to regular treatment) and microbiological evidence (Aspergillus isolation in LRT sample or two consecutive positive serum galactomannan [GM] tests). As for possible IPA, it required host factors but without microbiological evidence. Colonization was defined as isolation of Aspergillus in LRT samples without any symptom or new pulmonary infiltrate. In this study, Proven/probable IPA were taken as IPA; possible IPA with positive response to antifungal therapy were also considered as IPA.

\section{Risk factors}

Data were collected from eligible AECOPD patients concerning potential risk factors for IPA. All risk factors were readily accessible from early current and past medical history, including demographic data, comorbidities, pulmonary function, pharmacological history (including oral or intravenous corticosteroids in the last 3 months and use of broad-spectrum antibiotic longer than 10 days in the last month), mechanical ventilation at admission and previous history of acute exacerbation. Detailed data were shown in Table 1.

\section{Statistical analysis}

Statistical analysis was performed using statistical software SPSS (version 25.0, Chicago, IL, USA) and R software (version 3.5.2). Data were expressed as mean \pm standard deviation (SD) or median (interquartile [IQR]) for continuous variables, whereas categorical variables were summarized as counts (percentage). Differences between patients in training and validation set, and between patients with and without IPA in the training set were explored using $X^{2}$ or Fisher exact test for categorical variables, $t$ test for normally distributed continuous variables and Mann-Whitney $U$ test for abnormally distributed variables. Variables with $P<0.05$ in univariate analysis of training set were substituted into multivariate analysis. We then implemented multivariate logistic regression analysis based on backward stepwise likelihood-ratio method, setting a $P$ value $<0.05$ for the inclusion of variables. A nomogram of risk-predictive model for IPA was developed from the regression purposeful variable by library 'rms' in R [11]. Patients in the internal 
Table 1 Clinical characteristics of AECOPD inpatients in the training set and validation set

\begin{tabular}{|c|c|c|c|}
\hline \multirow[b]{2}{*}{ Characteristic } & \multicolumn{2}{|l|}{ No. $(\%)^{*}$} & \multirow[b]{2}{*}{$\mathbf{P}$} \\
\hline & $\begin{array}{l}\text { Training set } \\
(n=616)\end{array}$ & $\begin{array}{l}\text { Validation set } \\
(n=264)\end{array}$ & \\
\hline IPA & $59(9.6)$ & $24(9.1)$ & 0.821 \\
\hline \multicolumn{4}{|l|}{ Age, years } \\
\hline Mean \pm SD & $75 \pm 10.8$ & $75 \pm 10.6$ & 0.936 \\
\hline Gender male & $525(85.2)$ & $222(84.1)$ & 0.666 \\
\hline Smoking index $>400$ & $113(18.3)$ & $59(22.3)$ & 0.170 \\
\hline \multicolumn{4}{|l|}{ Comorbidities } \\
\hline Previous tuberculosis & $37(6)$ & $21(8)$ & 0.286 \\
\hline Lung cancer & $25(4.1)$ & $17(6.4)$ & 0.129 \\
\hline Bronchiectasis & $8(1.3)$ & $7(2.7)$ & 0.163 \\
\hline Asthma & $18(2.9)$ & $14(5.3)$ & 0.084 \\
\hline Lobectomy surgery & $13(2.1)$ & $10(3.8)$ & 0.153 \\
\hline Other solid tumor & $23(3.7)$ & $13(4.9)$ & 0.414 \\
\hline Hypertension & $297(48.2)$ & $123(46.6)$ & 0.659 \\
\hline Diabetes mellitus & $94(15.3)$ & $43(16.3)$ & 0.700 \\
\hline Congestive heart failure & $119(19.3)$ & $72(27.3)$ & 0.009 \\
\hline $\begin{array}{l}\text { Chronic and acute kidney } \\
\text { disease }\end{array}$ & $44(7.1)$ & $15(5.7)$ & 0.427 \\
\hline Advanced liver disease & $8(1.3)$ & $1(0.4)$ & 0.292 \\
\hline Connective tissue disease & $13(2.1)$ & $7(2.7)$ & 0.622 \\
\hline \multicolumn{4}{|l|}{ Laboratory results } \\
\hline Serum albumin $<30 \mathrm{~g} / \mathrm{L}^{\ddagger}$ & $151(24.5)$ & $70(26.5)$ & 0.530 \\
\hline GOLD III-IV & $409(66.4)$ & $177(67)$ & 0.852 \\
\hline Respiratory failure & $129(20.9)$ & $74(28)$ & 0.022 \\
\hline \multicolumn{4}{|l|}{ Co-infection } \\
\hline Lung bacterial infection & $100(16.2)$ & $54(20.5)$ & 0.131 \\
\hline Pulmonary tuberculosis & $12(19.5)$ & $4(1.6)$ & 0.788 \\
\hline \multicolumn{4}{|l|}{ Previous treatment } \\
\hline Inhale corticosteroids & $124(20.1)$ & $47(17.8)$ & 0.424 \\
\hline $\begin{array}{l}\text { Oral or intravenous corticos- } \\
\text { teroids }\end{array}$ & $59(9.6)$ & $36(13.6)$ & 0.075 \\
\hline Cytotoxic drug utility & $2(0.3)$ & $4(1.5)$ & 0.070 \\
\hline $\begin{array}{l}\text { Broad-spectrum antibi- } \\
\text { otic }>10 \text { days }\end{array}$ & $45(7.3)$ & $28(10.6)$ & 0.104 \\
\hline Invasive ventilator utility & $49(8)$ & $28(10.6)$ & 0.202 \\
\hline ICU admission 1 month prior & $54(8.8)$ & $39(14.8)$ & 0.008 \\
\hline $\begin{array}{l}\text { Hospital acute exacerbation } \geq 2 / \\
\text { year }\end{array}$ & $111(18)$ & $51(19.3)$ & 0.649 \\
\hline
\end{tabular}

IPA invasive pulmonary aspergillosis, GOLD global initiative for chronic obstructive lung disease, $I C U$ intensive care unit

*Values are presented as numbers and percentages, unless otherwise indicated

${ }^{\ddagger}$ This result was obtained from the hospital admission

${ }^{\S}$ The GOLD stage was obtained from the latest pulmonary function test within the last year

validation set were used for assessing the discrimination and calibration of the nomogram. The discriminative ability was measured using the area under the ROC curve (AUC), which known as the c-statistic. Calibration of the model was assessed by comparison of the predicted and observed probability of IPA [12]. The fit of the scoring model was evaluated by the Hosmer-Lemeshow goodness-of-fit test. Decision curve analysis was performed according to van Calster et al. to assess the clinical utility of the nomogram, using the library 'rmda (risk model decision analysis)' in R [13]. Unless stated otherwise, a two-tailed $P$ value $<0.05$ was considered statistically significant.

\section{Results}

We screened 1277 hospitalized patients primarily diagnosed as AECOPD. 397 patients were excluded for the following reasons: neutropenia $(\mathrm{n}=1)$; Aspergillus colonization $(\mathrm{n}=10)$; not real AECOPD $(\mathrm{n}=298)$; insufficient clinical data $(n=82) .83$ cases were diagnosed as IPA (9.4\%), with an average age of $75.2 \pm 10.7$ (40-101 years old). The training set was consisted of 616 patients (IPA group 59 cases, non-IPA group 557 cases), and the validation set was consisted of 264 patients (IPA group 24 cases, non-IPA group 240 cases). Among all the IPA patients, 13 cases were diagnosed with proven IPA, 60 cases with probable IPA (20 cases with positive culture results of Aspergillus in the LRT specimens, 25 cases with two consecutive positive serum GM and 3 cases for positive bronchoalveolar lavage fluid (BALF) GM tests, 10 cases with positive results in both sputum culture and serum GM, 2 cases for positive results in BALF GM tests and the sputum test), and 10 cases with possible IPA all had positive response to antifungal therapy. IPA group in the training set consisted of 8 patients with proven IPA, 42 patients with probable IPA and 9 patients with possible IPA. IPA group in the validation set consisted of 5 patients with proven IPA, 18 patients with probable IPA and 1 patient with possible IPA. The flow chart shows the strategy to identify the participants of the AECOPD cohort (Additional file 1: Figure S1). The demographic and clinical characteristics of patients in the training set and validation set are listed in Table 1. The IPA incidence was comparable between two data sets $(9.6 \%$ vs $9.1 \% ; P=$ $0.821)$.

The characteristics of AECOPD patients with and without IPA in training set were summarized in Table 2. There was no difference in each group of co-infection including pulmonary tuberculosis. But, two groups varied from each other in terms of treatment before or after patients admitted to the ward, such as oral or intravenous corticosteroids in the last 3 months, use of broad-spectrum antibiotic longer than 10 days in last month, invasive ventilator utility and ICU admission in previous 30 days. Table 2 showed that IPA group had a significant difference with non-IPA in terms of 8 factors, including hypertension, serum albumin $<30 \mathrm{~g} / \mathrm{L}$, lung function GOLD 
Table 2 Comparison of AECOPD inpatients with and without IPA in the training set

\begin{tabular}{|c|c|c|c|}
\hline \multirow[t]{2}{*}{ Characteristic } & \multicolumn{2}{|l|}{ No. $(\%)^{*}$} & \multirow[t]{2}{*}{$\mathbf{P}$} \\
\hline & IPA $(n=59)$ & Non-IPA $(n=557)$ & \\
\hline \multicolumn{4}{|l|}{ Age, years } \\
\hline Median (range) & $72(67,82)$ & $76(68,84)$ & 0.08 \\
\hline Male gender & $55(93.2)$ & $470(84.4)$ & 0.69 \\
\hline Smoking index $>400$ & $16(27.1)$ & $97(17.4)$ & 0.07 \\
\hline \multicolumn{4}{|l|}{ Comorbidities } \\
\hline Previous tuberculosis & $6(10.2)$ & $31(5.6)$ & 0.15 \\
\hline Lung cancer & $4(6.8)$ & $21(3.8)$ & 0.29 \\
\hline Bronchiectasis & $1(1.7)$ & $7(1.3)$ & 0.78 \\
\hline Asthma & $1(1.7)$ & $17(3.1)$ & 0.56 \\
\hline Lobectomy surgery & $1(1.7)$ & $12(2.2)$ & 0.82 \\
\hline Other solid tumor & $0(0)$ & $23(4.1)$ & 0.11 \\
\hline Hypertension & $21(35.6)$ & $276(49.6)$ & 0.04 \\
\hline Diabetes mellitus & $7(11.9)$ & $87(15.6)$ & 0.44 \\
\hline Congestive heart failure & $13(22)$ & $106(19)$ & 0.57 \\
\hline Chronic and acute kidney disease & $4(6.8)$ & $40(7.2)$ & 0.96 \\
\hline Advanced liver disease & $2(3.4)$ & $6(1.1)$ & 0.14 \\
\hline Connective tissue disease & $0(0)$ & $13(2.3)$ & 0.24 \\
\hline \multicolumn{4}{|l|}{ Laboratory results } \\
\hline Serum albumin $<30 \mathrm{~g} / \mathrm{L}$ & $29(49.2)$ & $122(21.9)$ & $<0.01$ \\
\hline GOLD III-IV & $56(94.9)$ & $353(63.4)$ & $<0.01$ \\
\hline Respiratory failure & $13(22)$ & $116(20.8)$ & 0.83 \\
\hline \multicolumn{4}{|l|}{ Co-infection } \\
\hline Lung bacterial infection & $10(16.9)$ & $90(16.2)$ & 0.88 \\
\hline Pulmonary tuberculosis & $0(0)$ & $12(2.2)$ & 0.25 \\
\hline \multicolumn{4}{|l|}{ Previous treatment } \\
\hline Inhale corticosteroids & $11(18.6)$ & $113(20.3)$ & 0.76 \\
\hline Oral or intravenous corticosteroids & $18(30.5)$ & $41(7.4)$ & $<0.01$ \\
\hline Cytotoxic drug utility & $0(0)$ & $2(0.4)$ & 0.64 \\
\hline Broad-spectrum antibiotic $>10$ days & $17(28.8)$ & $28(5)$ & $<0.01$ \\
\hline Invasive ventilator utility & $9(15.3)$ & $40(7.2)$ & 0.03 \\
\hline ICU admission 1 month prior & $10(16.9)$ & $44(7.9)$ & 0.02 \\
\hline Hospital acute exacerbation $\geq 2 /$ year & $20(33.9)$ & $91(16.3)$ & $<0.01$ \\
\hline
\end{tabular}

IPA invasive pulmonary aspergillosis, GOLD global initiative for chronic obstructive lung disease, ICU intensive care unit

*Values are presented as numbers and percentages, unless otherwise indicated

III-IV, use of broad-spectrum antibiotic longer than 10 days in last month and so on.

It's worth noting that oral or intravenous corticosteroids in the last 3 months before admission was of statistical significance between the IPA and non-IPA groups. Therefore, we examined the cumulative dose of corticosteroids during the patients' treatment 90 days before their admission and found that 59 out of 616 patients used corticosteroids systematically during the last 90 days. All corticosteroids cumulative dose was calculated on prednisone equivalent basis
(0.75 mg of dexamethasone, $4 \mathrm{mg}$ of methylprednisolone or $20 \mathrm{mg}$ of hydrocortisone is equivalent to 5 $\mathrm{mg}$ of prednisone). 18 out of 59 patients in IPA group, with a mean cumulative dose of prednisone $718 \mathrm{mg}$ (range, 120-2050 mg), 41 out of 59 patients in nonIPA group, with a mean cumulative dose of prednisone $307 \mathrm{mg}$ (range, $75-1800 \mathrm{mg}$ ) in this study. The ROC curve is shown in Additional file 1: Figure S2, the area under the ROC curve was 0.75 (95\% CI, $0.62-0.88$; P = 0.002 ). The cut-off cumulative dose of prednisone for the risk of IPA was $265 \mathrm{mg}$, with a sensitivity of $66.7 \%$ and specificity $75.6 \%$. 


\section{Nomogram development}

The multivariate logistic regression model considered 8 parameters with $\mathrm{P}$ value $<0.05$, including hypertension, serum albumin $<30 \mathrm{~g} / \mathrm{L}$, GOLD III-IV, oral or intravenous corticosteroids, use of broad-spectrum antibiotic longer than 10 days in last month, invasive ventilator utility, ICU admission 1 month previously and hospital acute exacerbation $\geq 2$ /year. Multivariate logistic analysis showed four independent risk-predictive factors for IPA: lung function GOLD III-IV, oral or intravenous corticosteroids (prednisone) $\geq 265 \mathrm{mg}$ in the last 3 months, use of broad-spectrum antibiotic longer than 10 days in last month, and serum albumin $<30 \mathrm{~g} / \mathrm{L}$ (Table 3). According to multivariate regression results, the nomogram was generated based on the contributed weights of factors in the training set to calculate the risk of IPA (Fig. 1). In the nomogram, each factor has a related score for its contribution to IPA.

\section{Discrimination and calibration}

The c-statistic of the nomogram was 0.8 (95\%CI, 0.740.86 ) in the training set data (Fig. 2, blue curve), and 0.79 (95\%CI, 0.68-0.90) in the validation set (Fig. 2, red curve). The calibration belt suggested that the nomogram had strong concordance performance in both the training and validation data sets (Fig. 3a, b). The 95\%CI region of GiViTI calibration belt did not cross the 45-degree diagonal bisector line both in two data sets $(P=0.722, P=$ 0.887 ; respectively). The model showed good fit between predicted and observed probabilities because the $P$ value for the Hosmer-Lemeshow test was both more than 0.05 in the training set and validation set $(P=0.69, P=0.70$; respectively).

\section{Decision curve analysis}

Decision curve analysis results of the risk nomogram in the training set and validation set were shown to

Table 3 Multivariate logistic regression for IPA in the training set

\begin{tabular}{|c|c|c|c|c|}
\hline Variable & $\beta$ coefficient & Wald & $\begin{array}{l}\text { OR } \\
(95 \% \mathrm{Cl})\end{array}$ & $P$ \\
\hline Serum albumin $<30 \mathrm{~g} / \mathrm{L}$ & 0.8 & 6.7 & $\begin{array}{l}2.23 \\
(1.22 \text { to } 4.1)\end{array}$ & 0.01 \\
\hline GOLD III-IV & 2.06 & 11.2 & $\begin{array}{l}7.87 \\
(2.35 \text { to } 26.35)\end{array}$ & 0.001 \\
\hline Dose $\geq 265 \mathrm{mg}^{*}$ & 2.34 & 20.48 & $\begin{array}{l}10.36 \\
\text { (3.76 to 28.53) }\end{array}$ & $<0.001$ \\
\hline Broad-spectrum antibiotic $>10$ days & 1.56 & 16.81 & $\begin{array}{l}4.77 \\
(2.26 \text { to } 10.07)\end{array}$ & $<0.001$ \\
\hline
\end{tabular}

OR odds ratio, $\mathrm{Cl}$ confidence interval, Ref reference variable, GOLD global initiative for chronic obstructive lung disease

* Oral or intravenous corticosteroids (equivalent prednisone) $\geq 265$ mg last 3 months

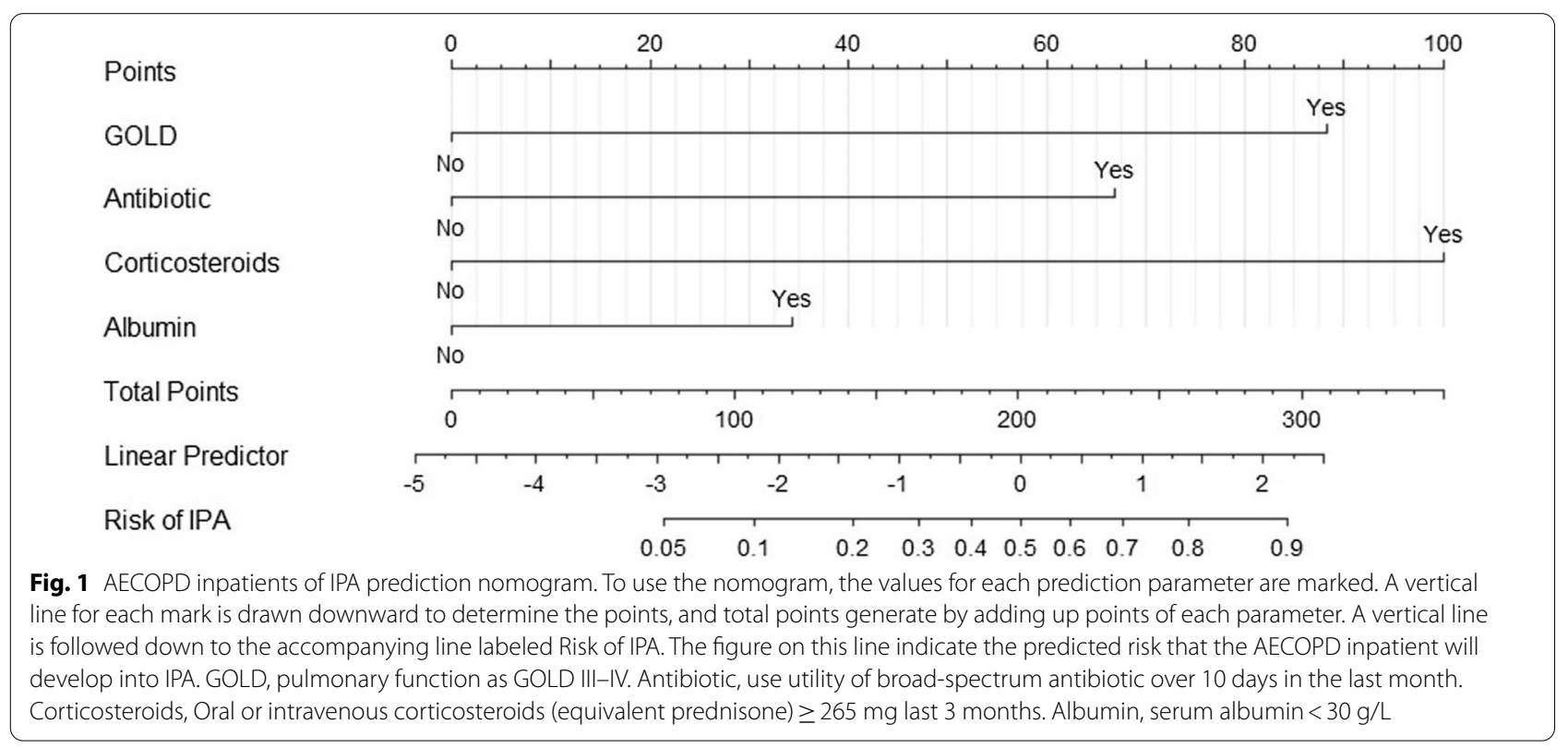




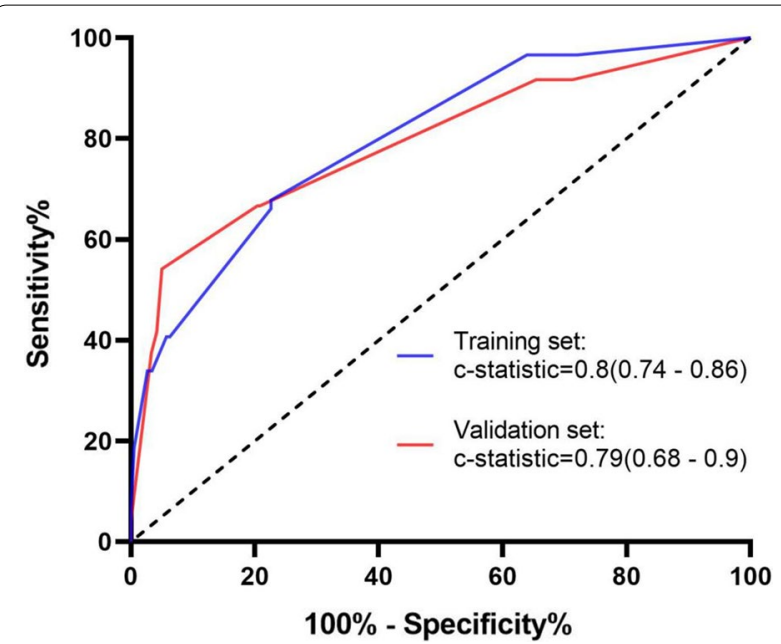

Fig. 2 Receiver operating characteristic (ROC) curves showing performance of the prediction model using both the training set and validation set $(P<0.0001$, vs 0.5$)$

determine an optimal decision point of the nomogram (Additional file 1: Figure S3). For predicted risk thresholds between 0 and 53\%, the nomogram model showed a positive net benefit in two data sets.

\section{Discussion}

In the real-world retrospective study, we developed and internally validated a multivariable model to identify AECOPD patients who are at risk for IPA. The prediction model based on easily accessible clinical parameters. Risk factors of predicting IPA in AECOPD patients comprised albumin, lung function and use of antibiotic and corticosteroids.

IPA in the context of COPD is attracting more and more attention. Current studies have reported that the incidence rate of IPA in COPD stands between 1.3 and $16.13 \%[7,14]$. This cohort study involved a large population of AECOPD patients, revealing the incidence and mortality of IPA, as well as the risk factors of developing IPA. Furthermore, our study shows a 9.4\% incidence of IPA in hospitalized AECOPD patients, which was at a relatively high level. Multiple reasons might lead to such a high incidence. First, our hospital locates in subtropical regions of Asia, where environmental fungi grow well due to the temperature and humidity, that might lead to a higher incidence of fungal infection. Second, patients suspected with pulmonary aspergillosis in these regions prefer to transfer to our department, which is a regional center for diagnosis and treatment of pulmonary mycosis.

Patients with AECOPD are often treated with corticosteroids. Current studies prove that corticosteroid is one of the independent risk factors for IPA in COPD [1], but there is no consensus on the cutoff value of accumulative dose of corticosteroids, especially in patients with COPD. Stuck et al, reported that corticosteroids rarely lead to a serious infection in patients with equivalent prednisone dose less than $10 \mathrm{mg} /$ day or a cumulative dose less than $700 \mathrm{mg}$ [15]. The EORTC/MSG Consensus Group definite prolonged use of prednisone at a mean minimum dose of $0.3 \mathrm{mg} / \mathrm{kg} /$ day for $>3$ weeks as a host factor for invasive fungal disease [16]. However, the study above was not focused on patients with COPD, and whether the cutoff value of cumulative dose of corticosteroids is

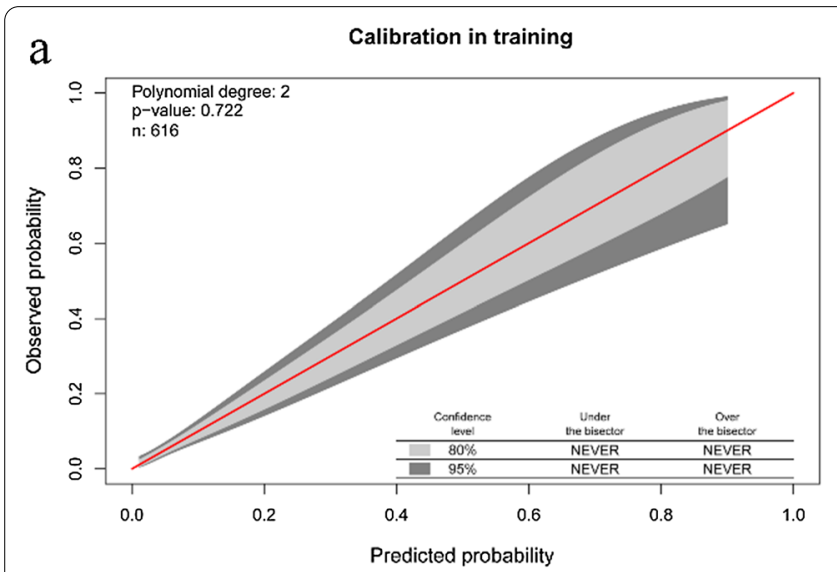

b

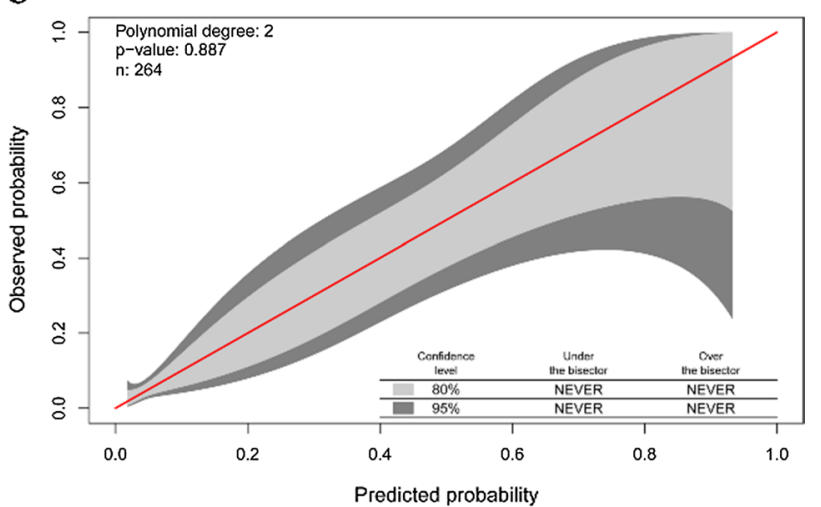

Fig. 3 Calibration belt comparing predicted probability of IPA from the nomogram and the observed probability of IPA. The 80\%- and 95\%-confidence level calibration belt are plotted, in light and dark grey respectively. The red diagonal line is the reference line, indicating the probability of an ideal nomogram. Calibration in training was calculated from training set data, and calibration in validation was calculated from validation set data 
suitable for COPD patients remained undetermined. Our study showed that the cumulative dose of systemic prednisone over $265 \mathrm{mg}$ in the last 3 months may lead to IPA in hospitalized AECOPD patients, with a sensitivity of $66.7 \%$ and specificity of $75.6 \%$. Our study indicates that inpatients with AECOPD may develop IPA even in a low dose of steroids. Besides, cases of IPA have even been reported in patients with long term inhaled high dose of steroids $[17,18]$.

The existing reports believed that early identification of IPA is essential for early IPA diagnosis and timely treatment $[19,20]$. In this regard, our study has made some clinical implications in the rapid identification of patients with high-risk IPA. The study also found that combined poor lung function (GOLD III-IV), serum albumin $<30 \mathrm{~g} / \mathrm{L}$ and use of broad-spectrum antibiotic longer than 10 days in last month are also independent risk factors for IPA in patients with AECOPD. To our best knowledge, we innovate to develop a simple nomogram for predicting risk of IPA in AECOPD patients. The good discriminative and calibrated ability of the predictive model is found in the internal validation cohort.

Three criteria have been proposed for diagnosing IPA. The criteria defined by the European Organization for Research and Treatment of Cancer/Mycosis Study Group (EORTC/MSG) were created for research in immunocompromised patients, but not good for mild immunocompromised or immunocompetent patients (like ICU or COPD patients) [16]. Clinical algorithm for ICU patients with Aspergillus-positive from lower respiratory tract (LRT) specimens demonstrated favorable validity to discriminate colonization from IPA [21]. The entry criterion of Aspergillus isolation from LRT specimens for clinical algorithm would result in missing diagnosis of probable IPA in ICU [22]. Diagnosis of IPA in our study was based on Bulpa criteria, which was proposed for patients with COPD [1]. Comparing with other two criteria, the Bulpa criteria yielded the highest diagnostic rate of probable IPA in critically ill COPD patients admitted to ICU [23]. Although available literature illustrated risk factors such as use of antibiotic or corticosteroids may predict IPA for COPD patients [7, 24], the accurate contribution and complex association of those factors to predict the likelihood of IPA has not been encompassed by the current criteria.

Nomograms are simple and visual prediction models by combining multiple indicators that can diagnose or predict diseases. The nomogram developed in this study may be useful for clinicians in evaluating the risk of COPD patients complicating IPA, further methods for diagnosing IPA will be actively pursued. For example, using the nomogram, an AECOPD patient with lung function of GOLD III-IV, use of broad-spectrum antibiotic over 10 days in the last month, systemic corticosteroids less than $265 \mathrm{mg}$ last 3 months and serum albumin more than $30 \mathrm{~g} / \mathrm{L}$, has a $26 \%$ predicted risk of IPA. One study showed that even GOLD II patients treated with steroids were at risk of developing IPA, but those patients would be missed according to Bulpa criteria [25]. An AECOPD patient with two or three risk factors in addition to lung function has $8-52 \%$ predicted risk of IPA calculated by the nomogram.

According to the EORTC/MSG and Bulpa criteria, IPA is categorized as proven, probable or possible. AECOPD patients with possible IPA who responded to antifungal therapy were included in this study. Possible IPA had no sufficient mycological evidence. They only performed the test of sputum culture and serum GM, while BALF GM and Aspergillus PCR were not performed. Sensitivity of serum GM is notably lower in non-neutropenic than neutropenic patients $[26,27]$. Compared to serum GM and LRT Aspergillus isolation, BALF GM had an excellent performance with higher sensitivity in diagnosis of IPA for COPD patients [28]. Meanwhile, clinical records showed possible IPA in our study received antifungal therapy before serum GM test, which would decrease the positive rate of serum GM.

Our study had some limitations. First, this study was a single-center retrospective study and we only did internal validation to evaluate the discrimination and calibration of the scoring model. Multicenter prospective studies should be conducted to externally validate the results. Second, the binary result (positive/negative) used to classify COPD co-morbidities cannot reflect the severity of the combined disease. Severity classifying of the comorbidities might add the value of the results.

In conclusion, the nomogram model, which is consisted of four independent risk factors for IPA, may empower clinicians and AECOPD patients with earlier, more accurate information regarding the risk of IPA. Further studies are needed to validate the application of the nomogram in clinical practice to determine whether IPA can be better predicted.

\footnotetext{
Abbreviations

IPA: Invasive pulmonary aspergillosis; COPD: Chronic obstructive pulmonary disease; AECOPD: Acute exacerbation of chronic obstructive pulmonary disease; ICU: Intensive care unit; GOLD: Global Initiative for Chronic Obstructive Lung Disease; LRT: Lower respiratory tract; GM: Galactomannan; SD: Standard deviation; IQR: Interquartile; AUC: Area under the ROC curve; BALF: Bronchoalveolar lavage fluid; EORTC/MSG: European Organization for Research and Treatment of Cancer and the Mycoses Study Group Education and Research Consortium.
} 


\section{Supplementary Information}

The online version contains supplementary material available at https://doi. org/10.1186/s12931-021-01771-3.

Additional file 1: Figure S1. Flow Chart for Training and Validation Patients Screening. Figure S2. Receiver operating characteristic (ROC) curve for cumulative dose of prednisone for the risk of IPA. Area under the ROC curve was $0.75(95 \% \mathrm{Cl}, 0.62-0.88) . P=0.002$. Figure S3. Decision curve analysis for the training set and validation set demonstrating the benefit for predicting clinically significant IPA for the nomogram. Dark grey line (All) is the net benefit of providing all AECOPD patients with IPA therapy. Black line (None) is the net benefit of providing no AECOPD patients with IPA therapy. The red curve indicates the net benefit provided by nomogram model in the training set and validation ser data. Table S1. TRIPOD Checklist.

\section{Acknowledgements}

Not applicable.

\section{Authors' contributions}

$X S, Y G, X Y$ and $Y L$ are the guarantor of the manuscript and take responsibility for the content of this manuscript. XS, YG, XY and $Y L$ contributed to the design of the study; $Y G, X Y, Y L, Y W$ and $K S$ were involved in the data analysis; $\mathrm{Yu} \mathrm{Gu}, \mathrm{XY}$ and $\mathrm{YL}$ wrote the initial draft of the manuscript, and the remaining authors were involved in revising the manuscript; $J Z$ and BC contributed to the acquisition of primary data; XS provided guidance for implementation and completion of the study. All authors read and approved the final manuscript.

\section{Funding}

This work was supported by the Project of Natural Science Foundation of China [Grant Numbers 82070011, 81873400], the Key Project of Jiangsu Commission of Health (K2019004), "333 project" of Jiangsu Province (BRA2019339), and Beijing Bethune Charitable Foundation (Grant Number BJ-RW2020004J).

\section{Availability of data and materials}

The datasets used and/or analyzed during the current study are available from the corresponding author on reasonable request.

\section{Declarations}

\section{Ethics approval and consent to participate}

The study protocol was approved by the Institute Ethics Committee of Jinling Hospital (2012NJKY-035-02). The study protocol conformed to the ethical guidelines of the 1975 Helsinki Declaration.

\section{Consent for publication}

Not applicable.

\section{Competing interests}

The authors declare that they have no competing interests.

\section{Author details}

'Department of Respiratory and Critical Care Medicine, Jinling Hospital, Nanjing Medical University, Nanjing 210002, China. ${ }^{2}$ Department of Medical Statistics, The First School of Clinical Medicine, Southern Medical University, Nanjing 210002, China. ${ }^{3}$ Department of Respiratory and Critical Care Medicine, Jinling Hospital, Medical School of Nanjing University, Nanjing 210002, China. ${ }^{4}$ Department of Respiratory and Critical Care Medicine, The First School of Clinical Medicine, Southern Medical University, Nanjing 210002, China.

Received: 18 February 2021 Accepted: 6 June 2021

Published online: 09 June 2021

\section{References}

1. Bulpa P, Dive A, Sibille Y. Invasive pulmonary aspergillosis in patients with chronic obstructive pulmonary disease. Eur Respir J. 2007;30:782-800.

2. Denning DW. The ambitious " $95-95$ by 2025 " roadmap for the diagnosis and management of fungal diseases. Thorax. 2015;70:613-4.

3. Ader F, Nseir S, Le Berre R, Leroy S, Tillie-Leblond I, Marquette CH, Durocher A. Invasive pulmonary aspergillosis in chronic obstructive pulmonary disease: an emerging fungal pathogen. Clin Microbiol Infect. 2005:11:427-9.

4. Patel DA, Gao X, Stephens JM, Forshag MS, Tarallo M. US hospital database analysis of invasive aspergillosis in the chronic obstructive pulmonary disease non-traditional host. J Med Econ. 2011;14:227-37.

5. Russo A, Falcone M, Vena A, Venditti C, Mancini C, Morelli A, Venditti M. Invasive pulmonary aspergillosis in non-neutropenic patients: analysis of a 14-month prospective clinical experience. J Chemother. 2011;23:290-4.

6. Xu H, Li L, Huang WJ, Wang LX, Li WF, Yuan WF. Invasive pulmonary aspergillosis in patients with chronic obstructive pulmonary disease: a case control study from China. Clin Microbiol Infect. 2012;18:403-8.

7. Guinea J, Torres-Narbona M, Gijon P, Munoz P, Pozo F, Pelaez T, de Miguel J, Bouza E. Pulmonary aspergillosis in patients with chronic obstructive pulmonary disease: incidence, risk factors, and outcome. Clin Microbiol Infect. 2010;16:870-7.

8. Benedict K, Richardson M, Vallabhaneni S, Jackson BR, Chiller T. Emerging issues, challenges, and changing epidemiology of fungal disease outbreaks. Lancet Infect Dis. 2017;17:e403-11.

9. Bassetti M, Righi E, De Pascale G, De Gaudio R, Giarratano A, Mazzei T, Morace G, Petrosillo N, Stefani S, Antonelli M. How to manage aspergillosis in non-neutropenic intensive care unit patients. Crit Care. 2014;18:458

10. Vestbo J, Hurd SS, Agusti AG, Jones PW, Vogelmeier C, Anzueto A Barnes PJ, Fabbri LM, Martinez FJ, Nishimura M, et al. Global strategy for the diagnosis, management, and prevention of chronic obstructive pulmonary disease: GOLD executive summary. Am J Respir Crit Care Med. 2013;187:347-65

11. Bursac Z, Gauss CH, Williams DK, Hosmer DW. Purposeful selection of variables in logistic regression. Source Code Biol Med. 2008;3:17.

12. Nattino G, Finazzi S, Bertolini G. A new test and graphical tool to assess the goodness of fit of logistic regression models. Stat Med. 2016;35:709-20.

13. Van Calster B, Wynants L, Verbeek JFM, Verbakel JY, Christodoulou E, Vickers AJ, Roobol MJ, Steyerberg EW. Reporting and interpreting decision curve analysis: a guide for investigators. Eur Urol. 2018;74:796-804.

14. Guinea J, Jensen J, Pelaez T, Gijon P, Alonso R, Rivera M, Munoz P, Bouza E. Value of a single galactomannan determination (Platelia) for the diagnosis of invasive aspergillosis in non-hematological patients with clinical isolation of Aspergillus spp. Med Mycol. 2008;46:575-9.

15. Stuck AE, Minder CE, Frey FJ. Risk of infectious complications in patients taking glucocorticosteroids. Rev Infect Dis. 1989;11:954-63.

16. Donnelly JP, Chen SC, Kauffman CA, Steinbach WJ, Baddley JW, Verweij PE, Clancy CJ, Wingard JR, Lockhart SR, Groll AH, et al. Revision and update of the consensus definitions of invasive fungal disease from the European Organization for Research and Treatment of Cancer and the Mycoses Study Group Education and Research Consortium. Clin Infect Dis. 2020;71:1367-76.

17. Palmer LB, Greenberg HE, Schiff MJ. Corticosteroid treatment as a risk factor for invasive aspergillosis in patients with lung disease. Thorax. 1991;46:15-20.

18. Leav BA, Fanburg B, Hadley S. Invasive pulmonary aspergillosis associated with high-dose inhaled fluticasone. N Engl J Med. 2000;343:586.

19. Schelenz S, Barnes RA, Barton RC, Cleverley JR, Lucas SB, Kibbler CC, Denning DW, British Society for Medical M. British Society for Medical Mycology best practice recommendations for the diagnosis of serious fungal diseases. Lancet Infect Dis. 2015;15:461-74.

20. Ullmann AJ, Aguado JM, Arikan-Akdagli S, Denning DW, Groll AH, Lagrou K, Lass-Florl C, Lewis RE, Munoz P, Verweij PE, et al. Diagnosis and management of Aspergillus diseases: executive summary of the 
2017 ESCMID-ECMM-ERS guideline. Clin Microbiol Infect. 2018;24(Suppl 1):e1-38.

21. Blot SI, Taccone FS, Van den Abeele AM, Bulpa P, Meersseman W, Brusselaers N, Dimopoulos G, Paiva JA, Misset B, Rello J, et al. A clinical algorithm to diagnose invasive pulmonary aspergillosis in critically ill patients. Am J Respir Crit Care Med. 2012;186:56-64.

22. Hamam J, Navellou JC, Bellanger AP, Bretagne S, Winiszewski H, Scherer E, Piton G, Millon L, Collaborative Rg. New clinical algorithm including fungal biomarkers to better diagnose probable invasive pulmonary aspergillosis in ICU. Ann Intensive Care. 2021;11:41.

23. Huang L, He H, Jin J, Zhan Q. Is Bulpa criteria suitable for the diagnosis of probable invasive pulmonary Aspergillosis in critically ill patients with chronic obstructive pulmonary disease? A comparative study with EORTC/ MSG and ICU criteria. BMC Infect Dis. 2017;17:209.

24. Delsuc C, Cottereau A, Frealle E, Bienvenu AL, Dessein R, Jarraud S, Dumitrescu O, Le Marechal M, Wallet F, Friggeri A, et al. Putative invasive pulmonary aspergillosis in critically ill patients with chronic obstructive pulmonary disease: a matched cohort study. Crit Care. 2015;19:421.

25. Bulpa P, Bihin B, Dimopoulos G, Taccone FS, Van den Abeele AM, Misset B, Meersseman W, Spapen H, Cardoso T, Charles PE, et al. Which algorithm diagnoses invasive pulmonary aspergillosis best in ICU patients with COPD? Eur Respir J. 2017. https://doi.org/10.1183/13993003.00532-2017.
26. Teering S, Verreth A, Peeters A, Van Regenmortel N, De Laet I, Schoonheydt K, Dits H, Van De Vyvere M, Libeer C, Meersseman W, Malbrain ML. Prognostic value of serum galactomannan in mixed ICU patients: a retrospective observational study. Anaesthesiol Intensive Ther. 2014:46:145-54.

27. Fortun J, Martin-Davila P, Gomez Garcia de la Pedrosa E, Silva JT, GarciaRodriguez J, Benito D, Venanzi E, Castano F, Fernandez-Ruiz M, Lazaro $F$, et al. Galactomannan in bronchoalveolar lavage fluid for diagnosis of invasive aspergillosis in non-hematological patients. J Infect. 2016;72:738-44.

28. He H, Ding L, Sun B, Li F, Zhan Q. Role of galactomannan determinations in bronchoalveolar lavage fluid samples from critically ill patients with chronic obstructive pulmonary disease for the diagnosis of invasive pulmonary aspergillosis: a prospective study. Crit Care. 2012;16:R138.

\section{Publisher's Note}

Springer Nature remains neutral with regard to jurisdictional claims in published maps and institutional affiliations.
Ready to submit your research? Choose BMC and benefit from:

- fast, convenient online submission

- thorough peer review by experienced researchers in your field

- rapid publication on acceptance

- support for research data, including large and complex data types

- gold Open Access which fosters wider collaboration and increased citations

- maximum visibility for your research: over $100 \mathrm{M}$ website views per year

At BMC, research is always in progress.

Learn more biomedcentral.com/submissions 